\title{
Angina secundaria a lesión de tronco coronario izquierdo post trasplante cardiaco. Evidencias de reinervación simpática tardía
}

\author{
Erick Riedemann $\mathbf{S}^{1}$, Pablo Castro $\mathbf{G}^{1}$, \\ Juan Carlos Q uintana F2, 0 svaldo Pérez P1, Paul Mc-Nab $M^{1}$. \\ Angina after a heart transplant \\ with evidence of late sympathetic \\ reinnervation. Report of one case
}

We report a 56 years-old man presenting with chest pain with exercise, seven years after an orthotopic heart transplant. A coronary angiography showed an atherosclerotic lesion in the common left main coronary artery with more than $90 \%$ obstruction. The lesion was successfully treated with a transluminal angioplasty with stenting. A 131-I metaiodobenylguanidine (MIBG) scan demonstrated sympathetic reinnervation. Sixteen months later, due to progression of allograft vasculopathy, coronary artery bypass was required (Rev Méd Chile 2007, 135: 1577-81).

(Key words: Coronary artery bypass; Coronary disease; Heart transplantation).

\author{
Recibido el 17 de agosto, 2006. Aceptado el 19 de marzo, 2007. \\ ${ }^{1}$ Departamento de Enfermedades Cardiovasculares y Cirugía Cardiaca. \\ ${ }^{2}$ Departamento de Radiología, Servicio de Medicina Nuclear \\ Hospital Clínico Pontificia Universidad Católica de Chile, Santiago de Chile.
}

\begin{abstract}
T a vasculopatía del injerto, también conocida Lcomo rechazo crónico, se refiere al desarrollo de enfermedad coronaria del corazón trasplantado y ocurre en $40 \%-70 \%$ de los receptores a 5 años ${ }^{1}$. Se diferencia de la enfermedad coronaria aterosclerótica habitual, en que las lesiones por rechazo crónico son generalmente difusas, distales y circunferenciales, ${ }^{2}$ lo que dificulta la revascularización en los casos de enfermedad coronaria avanzada. Como factores de riesgo para desarro-
\end{abstract}

$\overline{\text { Correspondencia a: Dr. Pablo Castro G. Departamento En- }}$ fermedades Cardiovasculares. Pontificia Universidad Católica de Chile. Marcoleta 347. Santiago Chile. Fax: 56-26392037. E mail: pcastro@med.puc.cl llar vasculopatía del injerto, se han descrito en el donante una mayor edad e hipertensión arterial, y en el receptor el sexo masculino, la raza negra y la infección por citomegalovirus. ${ }^{1}$ Debido a la denervación propia del miocardio trasplantado, la presentación clínica de la enfermedad coronaria es insuficiencia cardíaca, arritmias y muerte súbita, siendo poco frecuente la presencia de angina. ${ }^{3} \mathrm{La}$ existencia de angina sugiere reinervación miocárdica. ${ }^{4}$ Presentamos el caso de un paciente post trasplante cardíaco tardío, que se presenta con angina típica asociada a lesión de tronco coronario izquierdo (TCI), en el cual se evidencia reinervación cardíaca con cintigrama con $\mathrm{I}^{131}$ meta-yodo-bencil-guanidina (MIBG). 


\section{Caso ClínICO}

Hombre de 56 años, con antecedentes de miocardiopatía dilatada avanzada y trasplante cardíaco ortotópico biauricular 7 años atrás, procedente de un donante de 40 años de edad. Presentó un episodio de rechazo humoral al cuarto año post trasplante, que se trató exitosamente con metilprednisolona; en esa oportunidad la coronariografía fue normal. Tiene antecedentes de hipertensión arterial y diabetes mellitus adquiridas post trasplante. Se encontraba en tratamiento con azatioprina y ciclosporina, además de pravastatina, aspirina, enalapril y amlodipino. Ingresó por una historia de 2 meses de evolución de angina de esfuerzos moderados, asociado a disnea, que cedía con el reposo. Al examen físico, sólo destacaba una frecuencia cardíaca de 100 latidos por minuto y un cuarto ruido cardíaco. El electrocardiograma de reposo mostró ritmo sinusal regular, imagen de bloqueo completo de rama derecha con qS en V1, hemibloqueo izquierdo anterior y alteraciones inespecíficas de la repolarización ventricular en pared anterior. Exámenes de laboratorio: glicemia de $175 \mathrm{mg} / \mathrm{dL}$, creatininemia $1.29 \mathrm{mg} / \mathrm{dL}$, colesterol LDL $62 \mathrm{mg} / \mathrm{dL}$. Se realizó una biopsia endomiocárdica que fue negativa para rechazo celular. La ventriculografía angiográfica reveló una disfunción leve a moderada del ventrículo izquierdo e hipokinesia anteroapical. El cateterismo coronario mostró una lesión crítica ostial del TCI, la arteria descendente anterior se encontraba ocluida luego del nacimiento del primer ramo diagonal y se llenaba por heterocolaterales desde la arteria coronaria derecha. No se encontraron otras lesiones coronarias significativas. Luego de discutir las alternativas terapéuticas con el paciente se realizó angioplastia exitosa de la lesión del TCI, con implante directo de stent Taxus $^{\circledR} 3.5 \times 22 \mathrm{~mm}$ con flujo final TIMI 3 (Figura 1A y 1B). Un registro contínuo del segmento ST en doce derivaciones, detectó infradesnivel significativo y transitorio del segmento ST en la derivación V4 durante la angioplastia. No se registraron otros incidentes durante y después del procedimiento. Al séptimo día se realizó cintigrama con ${ }^{131}$ MIBG (Figura 2), demostrándose captación del radiofármaco en la pared anterior del ventrículo izquierdo. El pacien-

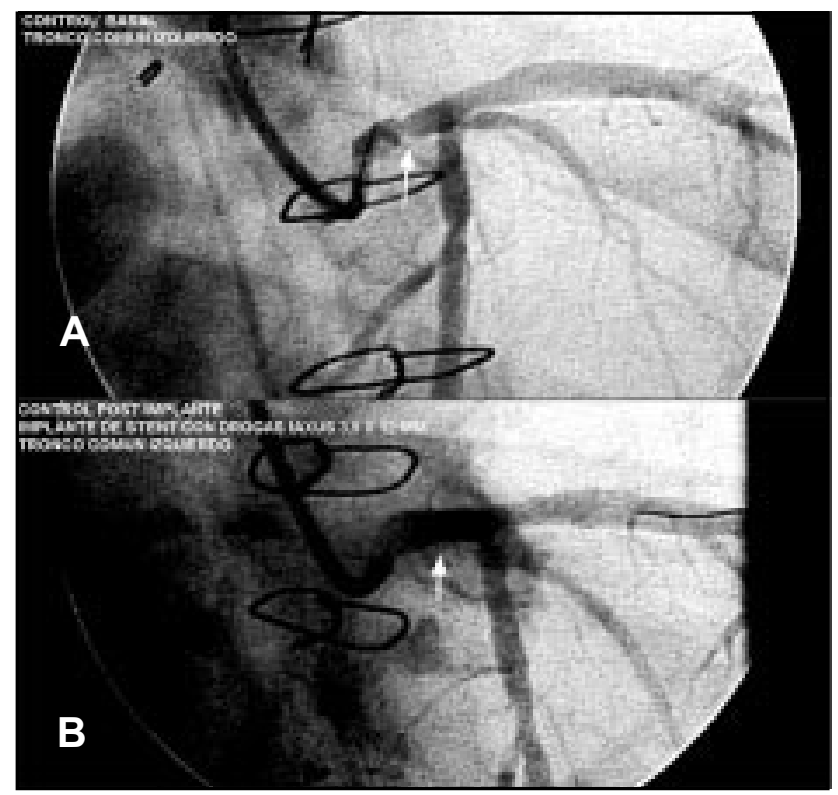

Figura 1. A. Lesión crítica ostial del TCI (flecha blanca) en coronariografía pre angioplastía transluminal. B. Coronariografía post angioplastía transluminal con implante de un stent Taxus $^{\circledR} 3.5$ x $22 \mathrm{~mm}$ con flujo final TIMI 3. 


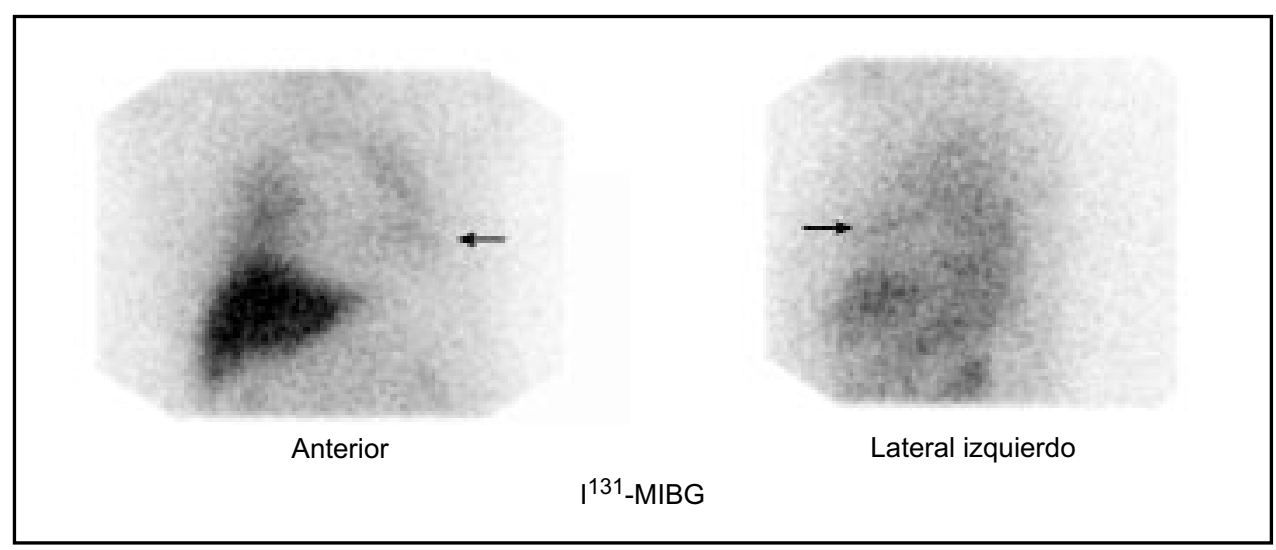

Figura 2. Imagen anterior y lateral izquierda de tórax con $\mathrm{I}^{131}$-MIBG obtenida 24 h post inyección. Se observa captación del radiofármaco en pared anterior del ventrículo izquierdo (flechas). No hay captación en el resto de las paredes. La distribución hepática del radiotrazador es normal. En sujetos normales puede haber captación pulmonar de intensidad variable, mayor en las bases.

te fue dado de alta 4 días post angioplastia en buenas condiciones, se incrementó la dosis de estatina (pravastatina $40 \mathrm{mg} /$ día) y se agregó clopidogrel a su terapia habitual. A los 16 meses post procedimiento, el paciente presentó disnea progresiva realizándose nueva coronariografía. Se constató ausencia de reestenosis angiográfica del TCI y progresión de la enfermedad aterosclerótica de las arterias circunfleja y coronaria derecha, las que presentaban estenosis significativas. Se realizó cirugía de revascularización miocárdica: injerto de arteria mamaria interna izquierda a arteria descendente anterior y puentes venosos safenos a arteria descendente anterior, rama posterolateral de la arteria circunfleja y coronaria derecha. El paciente evolucionó con bloqueo auriculoventricular postoperatorio que requirió del implante de marcapaso definitivo bicameral. Su evolución fue satisfactoria y fue dado de alta 14 días después de la cirugía de revascularización.

\section{DisCUSIÓN}

De nuestros registros de pacientes trasplantados cardíacos, este es el primer caso que se presenta con enfermedad coronaria aterosclerótica proximal, que se manifiesta con dolor anginoso, demostrándose reinervación simpática.

La reinervación simpática ocurre hasta en $67 \%$ a los 5 años del trasplante cardíaco y se ha asociado con la menor edad tanto del donante como del receptor, una cirugía de trasplante más rápida y con menos complicaciones y a receptores con baja frecuencia de rechazos. ${ }^{5}$ La reinervación simpática miocárdica puede ser demostrada utilizando radiotrazadores tales como $\mathrm{I}^{131}$ meta-iodobencil-guanidina $\left(\mathrm{I}^{131} \text {-MIBG }\right)^{7,12} \mathrm{O}$, más recientemente, con $\mathrm{C}^{11}$ hidroxiefedrina ( $\mathrm{C}^{11}$-HED). ${ }^{6}$ Estos radiofármacos se incorporan en las terminaciones simpáticas presinápticas postganglionares y no son catabolizados por la monoamino oxidasa ni por la catecol-O-metil-transferasa. La emisión gamma del $\mathrm{I}^{131}$ y la emisión de positrones del $\mathrm{C}^{11}$ permite obtener imágenes in vivo de las terminaciones simpáticas mediante cintigrafía ${ }^{13}$ y tomografía por emisión de positrones ${ }^{14}$ respectivamente. La reinervación simpática se inicia en la pared anterior y posteriormente se extiende al resto del miocardio.

Las alternativas de revascularización en el paciente trasplantado de corazón incluyen la angioplastia con stent y la revascularización quirúrgica. Los resultados de estos procedimientos son paliativos y en casos avanzados se plantea el retrasplante. La decisión de manejar una lesión de TCI con angioplastia con stent en vez de cirugía de revascularización se tomó luego de evaluar los riesgos de ambas alternativas y las preferencias del paciente. Además, existe una mayor morbimortalidad de la cirugía coronaria respecto de pacientes no trasplantados 9,10 . En este paciente en 
particular existía oclusión de un vaso con llene por colaterales e infarto antiguo en esa zona, y se decidió angioplastia del tronco con stent $^{8}$. En angioplastia con stent de corazones trasplantados, se ha reportado una tasa de reestenosis significativa a los 6 meses $(25 \%)^{15}$. Las tasas de reestenosis parecen ser mayores para las angioplastias que usaron stents metálicos (31\%) al compararlos con los stents con drogas (15\%-19\%) $)^{16,17}$. En cuanto a la angioplastia con stent del TCI en el paciente con corazón trasplantado la experiencia es limita-

\section{REFERENCIAS}

1. Costanzo M, Naftel D, Pritzker M, Heilman J 3rd, Boehmer J, Brozena S, eT AL. Heart transplant coronary artery disease detected by coronary angiography: a multinstitutional study of preoperative donor and recipient risk factors. Cardiac Transplant Research Database. I Heart Lung Transplant 1998; 17: 744-53

2. Tuzcu E, De Franco A, Goormastic M, Hobbs R, Rincon G, Bott-Silverman C, et al. Dichotomous pattern of coronary atherosclerosis 1 to 9 years after transplantation: insights from systematic intravascular ultrasound imaging. JACC 1996; 27 : 839-46.

3. Gao S, Schroeder J, Hunt S, Billingham M, ValantiNe H, Stinson E. Acute myocardial infarction in cardiac transplant recipients. Am J Cardiol 1989; 64: 1093-7.

4. Di Cori A, Petronio A, Gemignani C, Zucchelli G, Di Bello V, Mariani M. Symptomatic acute myocardial infarction in a cardiac transplant recipient successfully treated with primary coronary angioplasty: evidence of prognostic importance of chest pain after cardiac transplantation. I Heart Lung Transplant 2005; 24: 1146-9.

5. Bengel F, Ueberfuhr P, Hesse T, Schiepel N, Ziegler S, Scholz S, ET AL. Clinical determinants of ventricular sympathetic reinnervation after orthotopic heart transplantation. Circulation 2002; 106: 831-5.

6. Bengel F, Ueberfuhr P, Schiepel $\mathrm{N}$, Nekolla $\mathrm{S}$, Reichart B, Schwaiger M. Myocardial efficiency and sympathetic reinnervation after orthotopic heart transplantation: a noninvasive study with da requiriendo en la mayoría de ellos cirugía de revascularización posterior pese a uso de stent con drogas ${ }^{18,19}$.

En nuestro paciente, el procedimiento de angioplastia del TCI fue exitoso en el corto plazo. Posteriormente, debido a la progresión de la enfermedad aterosclerótica manifestada por insuficiencia cardiaca se decidió cirugía de revascularización miocárdica. La experiencia con esta última alternativa es limitada, describiéndose una elevada mortalidad?.

positron emission tomography. Circulation 2001; 103: 1881-6.

7. Momose M, Kobayashi $\mathrm{H}$, Ikegami $\mathrm{H}$, Matsuda $\mathrm{N}$, Hachida M, Kasanuki H, et al. Regional cardiac sympathetic reinnervation in transplanted human hearts detected by 123I-MIBG SPECT imaging. Ann Nucl Med 2000; 14: 333-7.

8. Benza R, Zoghbi G, Tallaj J, Brown R, Kirklin J, HubBard M, et al. Palliation of allograft vasculopathy with transluminal angioplasty. A decade of experience. JACC 2004; 43: 1973-81.

9. Halle A 3RD, DiSciascio, Massin E, Wilson R, Johnson M, Sullivan H, et Al. Coronary angioplasty, atherectomy and bypass surgery in cardiac transplant recipients. JACC 1995; 26: 120-8.

10. Musci M, Loebe M, Wellnhofer E, Meyer R, Pasic M, Hummel M, ET AL. Coronary angioplasty, bypass surgery, and retransplantation in cardiac transplant patients with graft coronary disease. Thorac Cardiovasc Surg 1998; 46: 268-74.

11. Hosenpud J, Bennett LE, Keck BM, Fiol B, Boucek $\mathrm{M}$, Novick R. The registry of the international society for heart and lung transplantation: sixteenth official report 1999. J Heart Lung Transplant 1999; 18: 611-26.

12. De Marco T, Dae M, Yuen-Green M, Kumar S, Sudhir K, KeIth F, ET AL. Iodine-123 metaiodobenzylguanidine scintigraphic assessment of the transplanted human heart: evidence for late reinnervation. JACC 1995; 25: 927-31.

13. Estorch M, Flotats A, Campreciós M, Marí C, Bernà L, Catafau A, et al. Reinervación simpática del corazón trasplantado. Estudio realizado con metayodobenzilguanidina marcada con yodo-123. Rev Esp Cardiol 1998; 51: 369-74. 
14. Schwaiger M, Hutchins G, Kalff V, Rosenspire K, Haka M, Mallette S, et al. Evidence for regional cathecolamine uptake and storage sites in the transplanted human heart by positron emission tomography. J Clin Invest 1991; 87: 1681-90.

15. Heublein B, Pethig K, MaAB C, Wahlers T, Haverich A ET AL. Coronary artery stenting in cardiac allograft vascular disease. Am Heart J 1997; 134: 930-8.

16. Tanaka K, Li H, Curran PJ, Takano Y, Arbit B, CURrier JW, ET AL. Usefulness and safety of percutaneous coronary interventions for cardiac transplant vasculopathy. Am J Cardiol 2006; 97: 1192-7.

17. Bader FM, Kfoury AG, Gilbert EM, Barry WH, Humayun N, Hagan ME, et al. Percutaneous coro- nary interventions with stents in cardiac transplant recipients. J Heart Lung Transplant 2006; 25: 298-301.

18. Gevigney G, Roriz R, MacFadden E, Boissonnat P, Chuzel M, Garé JP et al. Should we still perform angioplasty and stenting of an unprotected left main coronary artery stenosis in heart transplant patients? Two new cases and a review of the literature. I Heart Lung Transplant 2001; 20: 1217-9.

19. Matos G, Steen L, Leya F. Treatment of unprotected left main coronary artery stenosis with a drug eluting stent in a heart transplant patient with allograft vasculopathy. Heart 2005; 91: e11. 